\title{
Parity Indices and Two-Line Matrix Representation for Partitions
}

\author{
I.M. CRAVEIRO ${ }^{1 *}$, A. WAGNER ${ }^{2}$ and D. DOMINGUES ${ }^{3}$
}

Received on November 6, 2014 / Accepted on October 28, 2015

\begin{abstract}
In this work we present a solution for Andrews's Problem 5 [1] by establishing a bijection between the sets $D_{n}^{0}$ and $D_{n}^{1}$ defined in Fine's Theorem [8] and the sets of partitions indexed by their lower parity index [1]. We also solve Andrews's Problem 6, conjectured in [1].
\end{abstract}

Keywords: partition, parity index, mock theta function.

\section{INTRODUCTION}

In this section we present one of Fine's renowned theorems. Its combinatorial proof can be found in [8]. That proof does not use complicated analytic formulae and its popularization occurred because of its elegance and simplicity, being similar to the famous involutive proof by Franklin. Franklin's involution was used in many occasions to prove several refinements of Euler's Pentagonal Number Theorem [7].

Theorem 1.1 (Fine). Let $D_{n}^{0}$ and $D_{n}^{1}$ be the sets of partitions of $n$ into distinct parts, such that the largest part $a(\lambda)=\lambda_{1}$ is even and odd, respectively. Then

$$
\left|D_{n}^{0}\right|-\left|D_{n}^{1}\right|=\left\{\begin{aligned}
1, & \text { if } n=\frac{k(3 k+1)}{2} \\
-1, & \text { if } n=\frac{k(3 k-1)}{2} \\
0, & \text { otherwise. }
\end{aligned}\right.
$$

Problems that involve parity are related to classic partition identities such as Euler's, Rogers's, Ramanujan's and Gordon's identities. The theorem we just stated is an example of a problem that involves the concept of parity in the study of identities related to Partition Theory. Several authors

\footnotetext{
*Corresponding author: Irene Magalhães Craveiro.

${ }^{1}$ Faculdade de Ciências Exatas e Tecnologia - FACET, Universidade Federal de Grande Dourados - UFGD, Caixa Postal 364, 79084-970 Dourados, MS, Brasil. E-mail: irenecraveiro@ufgd.edu.br

2 DMA-IMECC, Universidade de Campinas - Unicamp, 13083-859 Campinas, SP, Brasil.

E-mail: dricawagner@gmail.com

${ }^{3}$ Centro de Ensino Monteiro de Carvalho, 20240-090 Santa Teresa, Rio de Janeiro, RJ, Brasil.

E-mail: deborarodrigues@gmail.com
} 
studied questions that involve parity in partitions, [4], [5], [6]. In [1], Andrews described several results on this subject. At the end of the same work he enumerated fifteen problems. Problems 1, 2 and 3 were solved in [10]. Problems 9 and 10 were solved in [12], with a purely analytic solution. In [11] other solutions for problems 9 and 10 are presented, and in the same paper a proof for problem 5 is given, which is based on some results related to generating functions for partitions and q-series. In [9] there is also a solution to problem 5, based on the involutive proof by Franklin, 1881.

In section 2 we present a solution to Problem 5 establishing a bijection between the sets $D_{n}^{0}$ and $D_{n}^{1}$ defined on Fine's Theorem and the sets of partitions indexed by their lower odd parity index [1]. In section 4 we present a solution for Problem 6, conjectured in [1].

We adopt the standard $q$-series notation: for each integer $n>0$ define

$$
(a)_{n}=(a ; q)_{n}=\prod_{j=0}^{n-1}\left(1-a q^{j}\right) \text { and }(a ; q)_{0}=1 .
$$

\section{LOWER INDEX AND FINE'S THEOREM}

Definition 2.1. Let $\lambda$ be a partition $\lambda=\lambda_{1}+\lambda_{2}+\cdots+\lambda_{j}$, where

$$
\lambda_{1} \geq \lambda_{2} \geq \cdots \geq \lambda_{j}>0 \text {. }
$$

The lower odd parity index of $\lambda$, denoted by $I_{l o}$, is defined as the maximum length of the subsequences of $\left\{\lambda_{1}, \lambda_{2}, \cdots, \lambda_{j}\right\}$ whose terms alternate in parity beginning with an odd $\lambda_{i}$.

Example. Consider $\lambda=8+7+7+6+5+4+4+2+2+1$ a partition of 46 . Thus, $I_{l o}(\lambda)=6$.

We will denote by $p_{o}(r, m, n)$ the number of partitions of $n$ in $m$ distinct parts with $I_{l o}(\lambda)$ equals to $r$. Besides, $P_{o}(y, x ; q)$ is the generating function for partitions $\lambda$ of $n$ into $m$ distinct parts with $I_{l o}(\lambda)=r$, that is,

$$
P_{o}(y, x ; q)=\sum_{r, m, n \geq 0} p_{o}(r, m, n) y^{r} x^{m} q^{n}
$$

The next result, given in [1], provides an explicit formula for $P_{o}(y, x ; q)$.

Theorem 2.1. The generating function for partitions enumerated by $p_{o}(r, m, n)$ is given by:

$$
P_{o}(y, x ; q)=\sum_{n \geq 0} \frac{x^{n} y^{n} q^{\frac{n(n+1)}{2}}\left(-\frac{q}{y}\right)_{n}}{\left(q^{2} ; q^{2}\right)_{n}} .
$$

Evaluating (2.1) and (2.2) in $y=-1$ and $x=1$ we have:

$$
P_{o}(-1,1 ; q)=\sum_{r, N \geq 0} p_{o}(r, m, N)(-1)^{r} q^{N}=\sum_{n \geq 0} \frac{(-1)^{n} q^{\frac{n(n+1)}{2}}(q ; q)_{n}}{\left(q^{2} ; q^{2}\right)_{n}} .
$$


Problem 5 in [1] asks for a combinatorial proof for:

$$
\sum_{r, m \geq 0} p_{o}(r, m, N)(-1)^{r}=\left\{\begin{aligned}
1, & \text { if } \quad N=n(3 n+1) / 2 \\
-1, & \text { if } \quad N=n(3 n+5) / 2+1 \\
0, & \text { otherwise } .
\end{aligned}\right.
$$

The following lemma is very useful to solve Problem 5.

Lemma 2.1. Consider $\lambda=\lambda_{1}+\lambda_{2}+\cdots+\lambda_{m}$ a partition of the positive integer $N$ in $m$ distinct parts, with $I_{\text {lo }}(\lambda)=r$ and $\lambda_{1}>\lambda_{2}>\cdots>\lambda_{m}$. Then $r$ is even (odd) if, and only if, $\lambda_{1}$ is even (odd).

Proof. Let $\lambda=\lambda_{1}+\lambda_{2}+\cdots+\lambda_{m}$ be a partition of the positive integer $N$ in $m$ distinct parts, with $I_{l o}(\lambda)=r, \lambda_{1}>\lambda_{2}>\cdots>\lambda_{m}$ and $r$ even. Hence there exists an increasing subsequence of $\left\{\lambda_{1}, \lambda_{2}, \ldots, \lambda_{m}\right\}$, whose length is equal to $r,\left(\lambda_{i+r-1}, \ldots \lambda_{i+1}, \lambda_{i}\right)$ with an odd $\lambda_{i+r-1}$. Because $\lambda_{i+r-1}$ is odd and $r$ is even, then $\lambda_{i}$ is even. In the same way, we can conclude that if $\lambda_{i}$ is even, then $r$ is even, because otherwise $\lambda_{i+r-1}$ would be even, a contradiction.

If $\lambda_{1}=\lambda_{i}$ then the proof is over. Suppose, then, that $\lambda_{1}>\lambda_{i}$.

For all $i>j$, we have that $\lambda_{j}$ is even. If $\lambda_{j}$ is odd we have one more term of the sequence that alternates parity with $\lambda_{i}$, which is even, and so the subsequence has length $r+1$. However $I_{l o}(\lambda)=r$. Therefore $\lambda_{j}$ is even. It follows from taking $j=1$ that $\lambda_{1}$ is even.

Conversely, suppose that $\lambda_{1}$ is even. Suppose by absurd that $I_{l o}(\lambda)=r$ with an odd $r$, then there exists a subsequence of $\lambda,\left(\lambda_{i+r-1}, \ldots \lambda_{i+1}, \lambda_{i}\right)$, with an odd $\lambda_{i+r-1}$, and $r$ being the maximum with this property. Hence $\lambda_{i}$ is odd. We have that $\lambda_{1} \neq \lambda_{i}$, because $\lambda_{1}$ is even. Since $\lambda_{i}$ must alternate parity with $\lambda_{1}$ we have that $I_{l o}(\lambda) \geq r+1$. Therefore $r$ if even. It is similar in the case $r$ is an odd number.

We will denote the set of partitions of a positive integer $N$, with distinct parts, whose lower odd parity index is $I_{l o}(\lambda)=r$ by: $\mathcal{P}_{N}^{r}$. When $r$ is even we use the notation $\mathcal{P}_{N}^{0}$, and when $r$ is odd, the notation $\mathcal{P}_{N}^{1}$.

It follows from Lemma 2.1 that given a partition $\lambda=\lambda_{1}+\lambda_{2}+\cdots+\lambda_{m}$ of the positive integer $N$ in $m$ distinct parts with $I_{l o}(\lambda)=r$ and $\lambda_{1}>\lambda_{2}>\cdots>\lambda_{m}$, then:

$$
\lambda \in \mathcal{P}_{N}^{0} \text { if, and only if, } \lambda \in D_{N}^{0} .
$$

In the same way, it follows from Lemma 2.1 that

$$
\lambda \in \mathcal{P}_{N}^{1} \text { if, and only if, } \lambda \in D_{N}^{1} .
$$

This result establishes a 1-1 correspondence between the sets defined on Fine's Theorem and the set of partitions $\lambda$ indexed by $I_{l o}(\lambda)=r$. So we can conclude that

$$
\left|D_{N}^{0}\right|=\sum_{r, m \geq 0 ; r \text { even }} p_{o}(r, m, N)(-1)^{r}
$$


and

$$
-\left|D_{N}^{1}\right|=\sum_{r, m \geq 0 ; r \text { odd }} p_{o}(r, m, N)(-1)^{r} .
$$

It follows from (2.4) and (2.5) that

$$
\sum_{r, m \geq 0} p_{o}(r, m, N)(-1)^{r}=\left|D_{N}^{0}\right|-\left|D_{N}^{1}\right| .
$$

Therefore it follows from Theorem 1.1 that

$$
\begin{aligned}
\sum_{r, m \geq 0} p_{o}(r, m, N)(-1)^{r} & =\left|D_{N}^{0}\right|-\left|D_{N}^{1}\right| \\
& =\left\{\begin{aligned}
1, & \text { if } N=n(3 n+1) / 2 \\
-1, & \text { if } N=n(3 n+5) / 2+1 \\
0, & \text { otherwise. }
\end{aligned}\right.
\end{aligned}
$$

Therefore we have another solution for Andrews's Problem 5, [1].

Example. Let $N=11,12, m$ denote the number of distinct parts of a partition $\lambda$ of $N$ and $r$ be its lower odd parity index.

Table 1: Distinct partitions of 11 and 12.

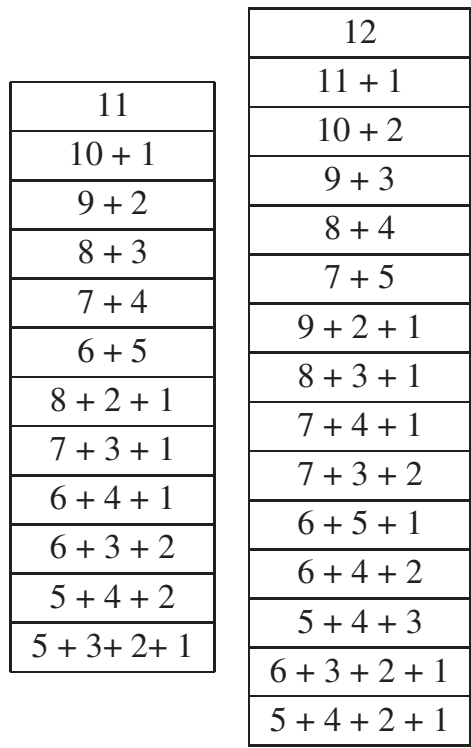

It follows from equation (2.7)

$$
\sum_{r, m \geq 0} p_{o}(r, m, 11)(-1)^{r}=0
$$


and

$$
\sum_{r, m \geq 0} p_{o}(r, m, 12)(-1)^{r}=-1 .
$$

In particular see cases $n=11$ and $n=12$, respectively in Table 2 .

Table 2: The values of $p_{o}(r, m, N)$, where $N=11$ and $N=12$, respectively.

\begin{tabular}{|c|c|c|c|}
\hline$m$ & $r$ & $p_{o}(r, m, 11)$ & $p_{o}(r, m, 11)(-1)^{r}$ \\
\hline 1 & 0 & 0 & 0 \\
\hline 1 & 1 & 1 & -1 \\
\hline 2 & 0 & 0 & 0 \\
\hline 2 & 1 & 2 & -2 \\
\hline 2 & 2 & 3 & 3 \\
\hline 3 & 0 & 0 & 0 \\
\hline 3 & 1 & 2 & -2 \\
\hline 3 & 2 & 3 & 3 \\
\hline 3 & 3 & 0 & 0 \\
\hline 4 & 0 & 0 & 0 \\
\hline 4 & 1 & 0 & 0 \\
\hline 4 & 2 & 0 & 0 \\
\hline 4 & 3 & 1 & -1 \\
\hline 4 & 4 & 0 & 0 \\
\hline
\end{tabular}

\begin{tabular}{|c|c|c|c|}
\hline$m$ & $r$ & $p_{o}(r, m, 12)$ & $p_{o}(r, m, 12)(-1)^{r}$ \\
\hline 1 & 0 & 1 & 1 \\
\hline 1 & 1 & 0 & 0 \\
\hline 2 & 0 & 2 & 2 \\
\hline 2 & 1 & 3 & -3 \\
\hline 2 & 2 & 0 & 0 \\
\hline 3 & 0 & 1 & 1 \\
\hline 3 & 1 & 1 & -1 \\
\hline 3 & 2 & 2 & 2 \\
\hline 3 & 3 & 3 & -3 \\
\hline 4 & 0 & 0 & 0 \\
\hline 4 & 1 & 0 & 0 \\
\hline 4 & 2 & 0 & 0 \\
\hline 4 & 3 & 1 & -1 \\
\hline 4 & 4 & 1 & 1 \\
\hline
\end{tabular}

\section{PROBLEM 6 GIVEN IN [1]}

The main goal of this section is to present a solution to Andrews's Problem 6, [1].

Definition 3.1. Given a partition $\lambda$ of a positive integer $n=\lambda_{1}+\lambda_{2}+\cdots+\lambda_{m}$ with $\lambda_{1} \geq$ $\lambda_{2} \geq \cdots \geq \lambda_{m}$, whose lower odd parity index equals to $I_{l o}(\lambda)=r$, the weight of $\lambda$, denoted by $w(\lambda)$ is the real number given by $(-1)^{r+m}$.

Lemma 3.1. Consider $\lambda=\lambda_{1}+\lambda_{2}+\cdots+\lambda_{m}$ a partition of the positive integer $N$, with $I_{l o}(\lambda)=r$ and $\lambda_{1} \geq \lambda_{2} \geq \cdots \geq \lambda_{m}$. Then: $r$ is even (odd) if, and only if, $\lambda_{1}$ is even (odd).

Proof. The proof of this lemma is similar to the proof of Lemma 2.1.

It follows from Lemma 3.1 that given any partition $\lambda$ of a positive integer $N$,

$$
w(\lambda)=(-1)^{m+r}=(-1)^{m+\lambda_{1}},
$$

where $m$ is the number of parts and $\lambda_{1}$ is its greatest part. 
In the following table we describe partitions $\lambda$ of 7 with its respectives $I_{l o}(\lambda)=r$, number of parts $m$ and weights $w(\lambda)$.

It follows from [1] that

$$
U_{o}(y, x ; q)=\sum_{r, m, n \geq 0} u_{o}(r, m, n) y^{r} x^{m} q^{n}
$$

is the generating function for the partitions $\lambda$ of the positive integer $n$ in exactly $m$ parts, with $I_{l o}(\lambda)=r$. Evaluating (3.1) in $x=y=-1$ we have:

$$
U_{o}(-1,-1 ; q)=\sum_{r, m, n \geq 0} u_{o}(r, m, n)(-1)^{m+r} q^{n}
$$

Observe that the coefficient of $q^{7}$ in (3.2) is given by $\sum_{r, m, \geq 0} u_{o}(r, m, 7)(-1)^{m+r}$. This is equivalent to sum the elements in the fourth column of Table 3 , which in this case equals to 7.

Table 3: Partitions of 7 with its respectives $I_{l o}(\lambda)=r$.

\begin{tabular}{|c|c|c|c|}
\hline partitions $\lambda$ of 7 & $I_{l o}(\lambda)=r$ & $m$ & $w(\lambda)=(-1)^{m+r}$ \\
\hline 7 & 1 & 1 & $(-1)^{1+1}=1$ \\
\hline $6+1$ & 2 & 2 & $(-1)^{2+2}=1$ \\
\hline $5+2$ & 1 & 2 & $(-1)^{1+2}=-1$ \\
\hline $5+1+1$ & 1 & 3 & $(-1)^{1+3}=1$ \\
\hline $4+3$ & 2 & 2 & $(-1)^{2+2}=1$ \\
\hline $4+2+1$ & 2 & 3 & $(-1)^{3+2}=-1$ \\
\hline $4+1+1+1$ & 2 & 4 & $(-1)^{4+2}=1$ \\
\hline $3+3+1$ & 1 & 3 & $(-1)^{3+1}=1$ \\
\hline $3+2+2$ & 1 & 3 & $(-1)^{3+1}=1$ \\
\hline $3+2+1+1$ & 3 & 4 & $(-1)^{4+3}=-1$ \\
\hline $3+1+1+1+1$ & 1 & 5 & $(-1)^{5+1}=1$ \\
\hline $2+2+2+1$ & 2 & 4 & $(-1)^{4+2}=1$ \\
\hline $2+2+1+1+1$ & 2 & 5 & $(-1)^{5+2}=-1$ \\
\hline $2+1+1+1+1+1$ & 2 & 6 & $(-1)^{6+2}=1$ \\
\hline $1+1+1+1+1+1+1$ & 1 & 7 & $(-1)^{7+1}=1$ \\
\hline
\end{tabular}

In [2] it is given a bijection between the set of unrestricted partitions and two-line matrices $A$ defined as:

$$
A=\left(\begin{array}{llll}
c_{1} & c_{2} & \ldots & c_{l} \\
d_{1} & d_{2} & \ldots & d_{l}
\end{array}\right)
$$


whose entries satisfy

$$
\left\{\begin{array}{c}
c_{l}>0 \\
c_{t} \geq 2+\quad c_{t+1}+d_{t+1} \quad \text { with } t<l
\end{array}\right.
$$

with $c_{t}, d_{t} \geq 0$ and $n=\sum_{i=1}^{l}\left(c_{i}+d_{i}\right)$. Matrices defined in this way are presented as:

$$
A=\left(\begin{array}{cccc}
(2 l-1)+j_{1}+\ldots j_{l}+d_{2}+\cdots+d_{l} & \ldots & 3+j_{l-1}+j_{l}+d_{l} & 1+j_{l} \\
d_{1} & \ldots & d_{l-1} & d_{l}
\end{array}\right),
$$

$l \geq 1, s, d_{1}, \ldots, d_{l}, j_{1}, j_{2}, \ldots, j_{l} \in \mathcal{N}$.

Given a partition $\lambda$ of a integer $n, n=\lambda_{1}+\lambda_{2}+\cdots+\lambda_{m}$, the procedure to obtain the matrix $A$ from $\lambda[2]$ is given below:

- the side $l$ of the Durfee square is the number of columns of the matrix;

- the parameters $d_{1}, d_{2}, \ldots, d_{l}$ indicate the amount of parts $1^{\prime} s, 2^{\prime} s, \ldots l^{\prime} s$, respectively, that appear below the Durfee square of side $l$.

$$
\left\{\begin{array}{c}
\lambda_{1}=l+j_{1}+j_{2}+\cdots+j_{l} \\
\vdots \\
\lambda_{l-1}=l+j_{l-1}+j_{l} \\
\lambda_{l}=l+j_{l}
\end{array},\right.
$$

where $j_{1}, j_{2}, \ldots, j_{l}$ are obtained in the solution of the system:

$$
\left\{\begin{array}{c}
c_{1}=(2 l-1)+j_{1}+j_{2}+\ldots j_{l}+d_{2}+\cdots+d_{l} \\
\vdots \\
c_{l-1}=3+j_{l-1}+j_{l}+d_{l} \\
c_{l}=l+j_{l}
\end{array}\right.
$$

In [3], the weight of the matrix $A$, given in (3.3) is defined by $w(A)=(-1)^{c_{1}+d_{1}+1}$. We will check that the weight of the matrix $A$ has the same weight of the partition $\lambda$, associated to $A$ by means of the bijection given in [3]. Indeed, $c_{1}+d_{1}+1=(2 l-1)+j_{1}+\ldots j_{l}+d_{2}+\cdots+d_{l}+d_{1}+$ $1=2 l+j_{1}+\ldots j_{l}+d_{1}+d_{2}+\cdots+d_{l}=\left(l+j_{1}+\ldots j_{l}\right)+\left(l+d_{1}+d_{2}+\cdots+d_{l}\right)$. Observe that the greatest part of the partition is $\lambda_{1}=l+j_{1}+\ldots j_{l}$ and the number of parts of the partition is: $m=l+d_{1}+d_{2}+\cdots+d_{l}$. Therefore, $w(A)=(-1)^{c_{1}+d_{1}+1}=(-1)^{\lambda_{1}+m}=(-1)^{r+m}=w(\lambda)$, where $r=I_{l o}(\lambda)$. It follows from [3] that

Theorem 3.1. The third order mock theta function $f(q)=\sum_{n=0}^{\infty} \frac{q^{n^{2}}}{(-q ; q)_{n}^{2}}$, is the generating function for the weighted number of matrices $A$ of the form (3.3). Where matrix is to be counted with the weight $w(A)=(-1)^{c_{1}+d_{1}+1}$.

With these observations and together with Theorem 3.1 we have: 
Theorem 3.2. The third order mock theta function $f(q)=\sum_{n=0}^{\infty} \frac{q^{n^{2}}}{(-q ; q)_{n}^{2}}$, is the generating function for the weighted number of unrestricted partitions $\lambda=\lambda_{1}+\lambda_{2}+\cdots+\lambda_{m}$ with $I_{l o}(\lambda)=r$. Where each partition is to be counted with the weight $w(\lambda)=(-1)^{m+r}$ and $m$ is the number of parts. From this from Theorem we have then, that

$$
U_{o}(-1,-1 ; q)=\sum_{r, m, n \geq 0} u_{o}(r, m, n)(-1)^{m+r} q^{n}=\sum_{n=0}^{\infty} \frac{q^{n^{2}}}{(-q ; q)_{n}^{2}}
$$

RESUMO. Neste trabalho apresentamos uma solução para o Problema 5 de Andrews [1] por meio de uma bijeção entre os conjuntos $D_{n}^{0}$ and $D_{n}^{1}$ definidos no Teorema de Fine [8] e os conjuntos das partições indexadas pelo índice de paridade inferior [1]. Também resolvemos o Problema 6 de Andrews, conjecturado em [1].

Palavras-chave: partição, índice de paridade, mock theta function.

\section{REFERENCES}

[1] G.E. Andrews. Parity in partition identities. Ramanujan Journal, 23 (2010), 45-90.

[2] E.H.M. Brietzke, J.P.O. Santos \& R. Silva. Bijective proofs using two-line matrix representation for partition. Ramanujan Journal, 23 (2010), 265-295.

[3] E.H.M. Brietzke, J.P.O. Santos \& R. Silva. Combinatorial interpretations as two-line array for the mock theta functions. Bulletin Brazilian Mathematical, 44 (2013), 233-253.

[4] H. Göllnitz. "Einfache Partionen". Thesis, Göttingen, (1960).

[5] H. Göllnitz. Partitionen mit Differenzenbedingungen. J. Reine Angew. Math., 225 (1967), 154-190.

[6] B. Gordon. Some continued fractions of the Rogers-Ramanujan type. Duke Math J., 31 (1965), 741-748.

[7] I. Martinjak. Refinements and Extensions of the Euler Partition theorem. http://imartinjak.files.wordpress.com/2013/06/refinementslecturenotes1.pdf.

[8] I. Pak. On Fine's partition theorems, Dyson, Andrews and missed opportunities. Mathematical Intelligencer, 25(1) (2003), p. 10.

[9] R. Silva, J.C. Filho \& J.P.O. Santos. Proving Two Partition Identities. TEMA, 13(2) (2012), 133-142.

[10] A.J. Yee \& S. Kim. Göllnitz-Gordon Identities and Parity Questions in Partitions. European Journal of Combinatorics, 32(2) (2011), 288-293.

[11] A.J. Yee. Ramanujan's partial theta series and parity in partitions. The Ramanujan Journal, 23 (2010), 215-225.

[12] C. Wenchang. Two Problems of George Andrews on Generating Functions for partitions. Miskolc Mathematical Notes, 13(2) (2012), 293-302. 